CHRONIQUE DE LA RECHERCHE

\title{
DU CONSENSUS DANS L'ECOLE HISTORIQUE FRANÇAISE
}

Quelques ouvrages récents donnent l'occasion d'effectuer un libre parcours à la recherche des positions de l'histoire dans les sciences sociales et la culture d'aujourd'hui. Il s'agit d'un bilan partiel (partial ?), assorti de quelques hypothèses et non, bien sûr, d'un tableau de la production historique. Ce bilan voudrait souligner les ambiguïtés des succès, des ambitions et de l'unité de l'école historique française et s'interroger sur les dangers du consensus qui semble y régner.

L'Histoire occupe une position privilégiée que traduit l'État des sciences sociales en France'. Après une introduction insistant sur l'éclatement de ce secteur, la présentation des différentes disciplines, des " pièces du puzzle ", pour reprendre le titre donné par les auteurs, commence par le chapitre " Histoire "... On sait l'importance de la première pièce du puzzle pour parvenir à la reconstruction de l'ensemble. Tout se passe comme si l'Histoire se voyait confier la tâche de ciment ou plutôt de garde-fou symbolique de l'unité des sciences sociales.

Cette fonction unificatrice impossible, l'Histoire la doit à deux éléments : elle recourt aux autres sciences sociales pour restituer un sens au passé - elle est donc infiniment contemporaine dans son outillage conceptuel et ses problématiques - et, en même temps, elle est, par le truchement de l'histoire des idées, suzeraine du passé des autres sciences sociales. Ce don d'ubiquité se trouve, par ailleurs, renforcé par un consensus sur la méthode historique traduit par un regroupement sous la bannière de l'école des Annales, j'allais écrire - plus justement? - par une inscription quasi obligatoire à la confrérie des Annales.

Plus l'histoire remplit son contrat d'être totale, plus elle couvre tous les domaines du social et plus elle investit les sciences voisines, tout en étant investie par elles, mais en évitant une perte d'identité grâce à une conscience de sa pratique très pragmatique : l'utilisation scientifique des sources.

1. L'État des sciences sociales en France, sous la dir. de Marc Guillaume, Paris, La Découverte, 1986.

Revue de synthèse: IV S. Nos 3-4, juil.-déc. 1988. 
Ainsi à l'extension de son domaine, à la complexité croissante de ses outils, l'historien oppose une identité salvatrice : la spécificité de sa pratique.

De ce que j'appellerai l'impérialisme protectionniste de l'Histoire, fondateur de sa position de force, le Dictionnaire des sciences historiques ${ }^{2}$ foumit une excellente illustration. Laissons la parole à André Burguière, le maitre-d'cuuvre de ce Dictionnaire :

"Ce dictionnaire n'a pas la prétention de les [autres synthèses sur l'Histoire] surpasser mais de les englober, de proposer un inventaire suffisamment large et approfondi des ressources intellectuelles d'une discipline qui est arrivée à un stade de maturité... mais non d'immobilité [...] les historiens ont pris place sur une multitude de secteurs spécialisés mais ils parlent un même langage [...] II règne chez les historiens [...] un large accord sur la définition et les exigences de la discipline; une situation qu'on ne retrouve pas forcément dans toutes les sciences de l'homme [...] [les débats et les querelles ont lieu] à l'intèrieur d'une même conception de ce qui fonde le caractère scientifique du travail de l'historien ${ }^{3}$.

On constate, dans cette déclaration d'intention, les fondements que je soulignais supra de l'aspect à la fois dominateur et sûr de lui du discours historique: il conquiert de nouveaux domaines sans douter de son unité ${ }^{4}$. Il fonctionne ainsi explicitement comme un centre durci par rapport à des périphéries plus molles.

Un aspect important de la cohésion de ce centre me parait être effectivement le langage. Les historiens sont génèralement lisibles les uns pour les autres et, gage d'un succès médiatique sur lequel nous reviendrons, le restent aussi pour le public cultivé. Cette heureuse tenue de langue des historiens s'enracine dans ses traditions littéraires et universitaires et leur garantit une plus grande immunité à l'encontre de la peste du jargon.

L'État des sciences sociales en fournit des preuves a contrario qui méritent une citation parmi, hèlas, tant d'autres. Précisons toutefois que l'amphigouri qui suit ne concerne pas toutes les autres présentations des sciences sociales, le chapitre consacrè à l'économie étant, entre autres, à la fois clair et pénétrant :

"L'unité épistémologique d'un champ de recherche dont la nomination [les sciences sociales] doit s'aider de deux identificateurs empruntés au langage commun fait question. On peut douter qu'il s'agisse ici d'une structure d'objets assez prégnante pour rendre solidaires paradigmes théoriques et méthodes d'investigation au point de faire sentir, d'un bout à l'autre du champ, les effets indivis des "revolutions scientifiques " ou du fonctionnement d'une « science normale » [...].

C'est aujourd'hui une vaste gamme d'intelligibilités partielles, indissociables d'un

2. Dictionnaire des sciences historiques, sous la dir. d'André Burguière, Paris, P.U.F., 1986. Souligné par moi.

3. Ibid., p. vil.

4. Certitudes et incertitudes de l'histoire, sous la dir. de Gilbert Gadoffre, Paris, P.U.F. (" Histoires "), 1987. Emmanuel Le Roy Ladurie remarque, p. 157 : " Il me semble que l'école historique française, malgré des divisions profondes, aboutit à un certain consensus [...]. Ce consensus bien sùr n'est pas toujours sain, j’en suis très conscient. " 
dispositif multi-dimensionnel de chantiers de recherche qui représente l'ensemble polysegmentaire de nos connaissances sur l'homme $[\ldots] \|^{5}$.

Sourire ne suffit pourtant pas. On constate dans ce passage que, pour exprimer une évidence, "les sciences sociales sont très hétérogènes et le terme qui les recouvre n'est guère satisfaisant ", on recourt à des circonvolutions. On comprend pourquoi : l'évidence contient une difficulté majeure - une situation d'éclatement - que l'on comble par une logorrhée. L'Histoire, au contraire, comble les trous du " champ social", non par un discours théorique inopérant en l'occurrence, mais par des significations scientifiquement établies. Cette impuissance du discours « épistémologico-sociologique " est bien traduite par l'opposition vide entre les termes abstraits employés et les termes concrets (objets, prégnant, champ, dispositif, chantier, etc.) qui ont une pure valeur incantatoire. Les tics du jargon des sciences sociales obéissent à une logique assez simple : gonfler le problème théorique par une inflation de termes abstraits et donner l'illusion de le dégonfler par un contrepoids de termes concrets. L'esprit est ainsi ballotté entre deux registres pléonastiques et enfermé, presque matériellement, entre deux miroirs sans tain laissant voir la même insoluble complexité.

Si l'on accorde une unité - relative - à l'Histoire appuyée sur celle des méthodes et du style, on peut être surpris que cette unité résiste aux coupures idéologiques. André Burguière place, on s'en souvient, débats, querelles et désaccords à l'intérieur du consensus alors que ce dernier terme s'accommode peu souvent des trois premiers ${ }^{6}$.

Pour saisir l'aspect officiellement familial des discordes idéologiques entre historiens, il faut souligner le rôle d'alma mater de "l'école des Annales". Le bel ouvrage consacré par Pierre Nora à la nation ${ }^{7}$ comporte, à juste titre, un chapitre historiographique ${ }^{8}$. Après l'évocation des grands ancêtres, sonne, dans le dernier article, "l'heure des Annales" ${ }^{9}$. Le titre est significatif dans sa volonté de souligner un moment déterminé, privilégié et fondateur d'un nouveau temps historique. A l'heure des Annales, chaque historien réglera désormais sa montre. Le Dictionnaire d'André Burguière est également un monument à la gloire de "l'école des Annales". Il ne s'agit évidemment pas de lui contester cette gloire ni d'oublier les fortes nuances apportées par les auteurs à l'unité d'une école qui participe plus d'un état d'esprit que d'un quelconque discours normatif. Néanmoins, et quitte à paraitre " surdéterminé " par l'appartenance au Centre international de synthèse, osons quelques remarques. A l'article «Annales» du Dictionnaire, A. Burguière présente « la revue

5. Cf. Jean-Claude PASSERon, "Les sciences sociales : unité et diversité », in op. cit. supra, n. 1, p. 10-11.

6. Dans le Dictionnaire d'A. BuRguière ne figure d'ailleurs pas d'entrée « Idéologie ».

7. Les Lieux de mémoire. La Nation, sous la dir. de Pierre NoRA, 3 vols, Paris, P.U.F., 1986.

8. L'actualité du livre témoigne sans cesse du dynamisme des recherches historiographiques. Il faut citer ici la très bonne étude de Jean WALCH, Les Maîtres de l'histoire, 18151850, Paris/Genève, Champion-Slatkine, 1986.

9. Cf. Krzysztof Pomian, “L'heure des Annales ", in op. cit. supra, n. 7, vol. 1, p. 377 429. 
fondée en 1929 [...] pour favoriser les contacts interdisciplinaires au sein des sciences sociales ${ }^{10}$, formule qui semble tout autant adaptée à la Revue de synthèse historique en... 1900. Même si la suite de l'article cite Henri Berr, il y a une volonté de présenter les Annales comme une rupture et l'ouverture d'une nouvelle ère qui me semble plus participer de la nécessité symbolique d'une origine, une année 0 d'un nouvel âge historique, que d'une réalité historiographique beaucoup plus évolutive. L'événement de 1929 n'échappe pas aux lois de la longue durée! L'article consacré à Henri Berr par O. Dumoulin" contient quelques injustices, "l'Évolution de l'Humanité " est plus qu'un " simple reflet de l'establishment universitaire " (sauf à considérer que les Annales en sont un autre); et, surtout, dire du Centre international de synthèse qu' « il est aussi un lieu de mondanités bien éloignées des ambitions intellectuelles originelles ", c'est commettre un contresens global sur le fonctionnement des milieux intellectuels au $\mathrm{xx}^{e}$ siècle. Dire qu'aujourd'hui nombre de nos grands historiens participent aux mondanités ne saurait en rien diminuer leur rôle intellectuel.

Laissons là le plaidoyer pro domo et étudions le rapport de l'école des Annales à l'idéologie. Comme le note le Dictionnaire, elle entretient des rapports certes ambivalents et évolutifs mais néanmoins certains avec le marxisme. A. Burguière souligne combien l'évolution de l'école des Annales par rapport aux autres sciences sociales suit celle du marxisme contemporain comme fleuve dans sa vallée (qui n'a rien d'une gorge, cependant) avec trois temps marquants : les rapports privilégiés avec la géographie, puis l'économie, puis la sociologie et l'anthropologie.

Dans son ouvrage décapant ${ }^{12}$, Alain Caillé va beaucoup plus loin en montrant la parenté avec le marxisme des Annales en général et de Fernand Braudel en particulier sur deux points clefs : la conception du marché, perçu comme éternel à l'opposé d'un capitalisme récent et pervers ${ }^{13}$, et la conception du temps. Braudel compare, en effet, « le présent de la période non à son présent propre ou à son passé, mais à son avenir. Autrement dit, avec Braudel, nous nous trouvons confrontés à une histoire implicitement rétrodictive, qui décide de l'importance relative des événements ou des institutions en fonction de ce qu'elle connait de leur devenir ultérieur et de leur signification actuelle ${ }^{14}$, conception inhérente au projet de Marx. Cette conception "rétrodictive" se trouve confirmée, a contrario, par l'évacuation dans le Dictionnaire du concept dès lors gênant de "Décadence " par un article en trompe-l'œil ${ }^{15}$ qui lui nie toute valeur heuristique et le juge disqualifié, insistant par-là mème sur la marginalité de Pierre Chaunu dans la famille historienne.

On voit, dès lors, comment cette famille se préserve des risques idéologiques

10. Cf. op. cit. supra, n. 2 , p. 47.

11. Ibid., p. 85-86.

12. Alain CAILlE, Splendeurs et misères des sciences sociales, Genève/Paris, Droz, 1986.

13. Ibid., p. 166.

14. Ibid., p. 167.

15. Cf. op. cit. supra, n. 2, p. 184. 
par un mariage intellectuel avec le marxisme. Non pas, bien sûr, un marxisme militant, non pas - sauf pour les plus évidemment marxistes des historiens, finalement minoritaires - le Marx de la conscience de classe et de l'action révolutionnaire mais le Marx de l'analyse de la production.

Le consensus des historiens est un consensus via la bannière des Annales, elle-même indemne de violences idéologiques centrifuges car liée intellectuellement au marxisme. Cette rencontre s'explique, bien sûr, par le succès du marxisme auprès des intellectuels jusque dans les années 60 mais aussi par la capacité du marxisme à définir des paradigmes et des modèles de nature authentiquement scientifiques. D'une scientificité perçue comme indispensable à une École centrée sur l'économique et le social. On voit comment la séduction s'opère à partir des noyaux durs - les moins polémiques, les plus scientifiques - du domaine historique du $\mathrm{xx}^{e}$ siècle et du marxisme.

Les années 70 et 80 marquées par un déclin du marxisme et une extension des pôles d'intérêt de l'école des Annales de l'économique vers le culturel ne changent pourtant pas radicalement la situation. Les rapports privilégiés entretenus dès lors entre Histoire et sociologie (et anthropologie) introduisent ce qu'on pourrait qualifier de pensée néo-marxiste de critique de "la classe dominante ", de l'ordre - nécessairement répressif - et des valeurs universelles.

L'ceuvre de Foucault est une balise commode de ces nouvelles modes intellectuelles de relativisation de la raison classique. En liaison avec le courant " libertaire " des années 60 , toute une série de recherches historiques s'engagent alors dans la description des marginalités de comportements comme de pensées et déplacent le centre de gravité des sociétés vers leurs exclus. On lira dans le dernier ouvrage de Raymond Boudon ${ }^{16}$ une heureuse remise en place de ces nouvelles idées reçues par la mise en valeur du fonctionnement a-scientifique des théories de Michel Foucault et de Pierre Bourdieu, par exemple.

L'histoire totale tend alors, du moins quant au volume de ses productions, à déséquilibrer le passé dans un émiettement de la culture en cultures juxtaposées. On n'a sans doute pas suffisamment mesuré combien le message initial de Lucien Febvre s'en trouve trahi. Celui-ci concevait, en effet, l'histoire des mentalités sous l'angle de catégories universelles (l'incroyance, la sensibilité ${ }^{17}$ ) dans la droite ligne du rationalisme classique. Les variations de ces catégories enregistrées par l'historien ne mettaient pas en cause leur valeur d'une universalité constitutive de l'honnête homme, d'un homme-sujet à part entière du processus culturel de l'humanité, en quête perpétuelle de sa signification. Lucien Febvre a été ainsi " trahi " à deux reprises par les préoccupations essentiellement " économiques et sociales " de ses contemporains " marxisants " puis par un (faux) retour à une histoire de la culture dont on ne croyait plus à l'unité et à l'universalité.

Au contraire de la perspective de Febvre, toute une partie de $œ$ qu'il faut

16. Raymond Boudon, L'Idéologie, l'origine des idées reçes, Paris, Fayard (« IdéesForces $)$ ), 1986.

17. On aura plaisir à relire ces textes dans La Sensibilité dans l'histoire, de Roger Chartier, Georges Duby, Lucien Febvre, Pierre Francastel, Robert Mandrou, SaintPierre-de-Saleme, Gérard Monfort, 1987. 
bien appeler la mode historique restitue, en effet, un homme en miettes, une vision de notre société sous l'angle de cultures ethniques juxtaposées qui fait de la marge le critère de vérité et qui se focalise sur les particularismes au détriment de tout universalisme.

Il y aurait ici une étude plus précise à faire sur " l'effet 68 " dans la vision du passé qui mettrait en évidence, derrière l'idée trompeuse d'histoire totale, une incapacité, ou mieux un refus, de dire l'unité du sujet et de la culture humaine. L'origine historique ne donne plus à lire un processus, une Bildung, comme chez Lucien Febvre, mais des particularismes irréductibles. Sans doute par pudeur, on ne signale guère combien cette " histoire des mentalités particulières " rejoint la tradition de la petite histoire anecdotique à la Lenôtre. La vie intime de Napoléon ou les états d'âme d'une domestique du XIX siècle : les deux approches me semblent également limitées et également flatteuses pour les paresses du grand public cultivé. Ce retour en force de l'anecdotique (futile quand il concerne les grands hommes, significatif quand il se rapporte aux masses), a d'ailleurs préparé le terrain à celui de la biographie.

Déplaçons un instant l'analyse du côté du consommateur d'histoire. Le succès médiatique de l'histoire se conçoit aisément : l'homme de cette fin de $\mathrm{xx}^{\mathrm{e}}$ siècle connaît une accélération du temps qui, confrontée à une vie humaine plus longue, l'oblige à affronter des bouleversements - techniques, professionnels, culturels - de plus en plus nombreux et profonds. D'autant plus qu'à cette accélération du temps correspond une dilatation de l'espace, de l'espace vécu par la révolution des transports comme de l'espace connu par la mondialisation de l'information. D'où cette fringale de passé, chargée de conjurer le brouillage des repères traditionnels du temps et de l'espace familiers. Or l'histoire elle-même ne saurait échapper à ce flux de l'expérience historique et reste aujourd'hui impuissante à restituer ces repères solides, cette totalité perdue. Rien de plus significatif à cet égard que l'ouvrage de Pierre Nora sur la nation; à une interrogation contemporaine sur ce qu'est devenue l'idée nationale, les historiens répondent par le repérage de significations effacées, par l'histoire d'une mémoire qui n'est plus.

$\mathrm{Au}$ lecteur avide de sens, l'historien ne peut restituer que des significations éparses et non hiérarchisées, et trop souvent anecdotiques, faute de répondre à la question des valeurs, et donc précisément de la hiérarchie dans la culture.

Le dernier ouvrage de Fernand Braudel ${ }^{18}$ me parait avoir posé de façon pathétique ce rapport au sens et au temps qui passe.

Sa volonté d'être donneur de sens éclate dès le titre, promesse de réponse altière à ce qui est devenue une interrogation éminemment actuelle : l'identité de la France. Elle apparait aussi dans le ton, que seul son statut scientifique permet à Braudel d'employer. Dès la première page, le maître restitue sa place à l'intimisme, l'historien saisi par la gageure de son sujet doit redevenir écrivain : « j’aime la France avec la même passion, exigeante et compliquée que Jules Michelet ", et il ajoute ce regret de devoir, le plus souvent, parler en historien,

18. Fernand Braudel, L'Identité de la France, 3 vols, Paris, Fammarion, 1986. 
de se condamner à "l'exclusion du coeur». Or, cette sécheresse, à laquelle Braudel veut s'astreindre, ne se retrouve pas dans l'ouvrage qui manifeste, au contraire, un discours historique comme ravissement nostalgique, comme une saisie d'épaisseur temporelle ${ }^{19}$, sensorielle et humaine, qui laisse place au rève, au " si » (si la France avait choisi la mer plutôt que la terre; si Rouen avait remplacé Paris, etc.).

Le plus saisissant est peut-être ailleurs. Tout se passe comme si Braudel percevait au fil de son écriture combien son objet lui échappe. Le projet est évidemment de comprendre l'identité présente par la longue durée. Mais, souvent, au cours des chapitres, le temps long, immortel, vient pourtant mourir sur la rapidité du présent, et l'histoire rétrodictive, dont nous parlions plus haut, perd ses repères actuels. L'identité de la France, patiemment saisie, apparait souvent et déjà comme une identité d'hier. Parmi tant d'autres, voyez ce passage où le regret et le doute l'emportent sur la conviction :

"La France est encore, pour un temps au moins, cet étagement du moins vite au très vite, au plus vite. Le plus vite, si brillant, si menacant soit-il, n'est pas encore tout. Alors oui, quel plaisir, seul dans une marche de montagne, de retrouver, de revivre, comme en écoutant le berger des pentes du mont Lozère, le temps et l'espace d'hier! " 20

Passage exemplaire, passage pathétique où l'on voit la longue durée et son historien comme réfugiés sur les hauteurs et voyant monter le bruit du présent menaçant.

Dans cet échec se mesure le bouleversement du rapport présent-avenir qui a, malgré lui, frappé Braudel dans son dernier ouvrage et l'a conduit à recourir au «je», à redonner à la conscience sa place, à humaniser la réponse que devaient apporter les structures.

Peut-on apporter une solution à cette suite de paradoxes, à ce bilan volontairement provocant? Elle réside peut-être dans la conscience que l'histoire totale n'est qu'une histoire éclatée et donc impuissante à penser l'articulation - plus difficile, voire douloureuse, que jamais - du présent et de l'avenir de nos sociétés. Une piste serait peut-être de lui redonner une unité en développant une nouvelle histoire des idées ${ }^{2 !}$, une histoire de la culture, et

19. Rappelons sur l'histoire du temps, l'ouvrage novateur et pénétrant de K. POMIAN, L'Ordre du temps. Paris, Gallimard ("Bibliothèque des histoires "), 1984.

20. Cf. F. BRAUDEL, op. cit. supra, n. 18, vol. 1, p. 107. Souligné par moi.

21. Cf. op. cit. supra, n. 4, p. 160-161. E. Le Roy Ladurie constate sur le mode de l'évidence tranquille: " Il y a bien sûr un déclin de l'histoire des idées au sens strict du terme, en tout cas une dévalorisation de cette discipline chez les historiens français du moins, dont l'intérêt s'est porté davantage vers les masses que vers l'élite, vers l'incarnation sociale - voire plébéienne - des idées plutôt que vers les produits intellectuels des grands penseurs [...] [il faut, pour intéresser les historiens français, que les idées], comme on disait en style marxiste autrefois, deviennent une force matérielle en pénétrant les masses. "C'est à cette évidence consensuelle que nous ne saurions précisément nous résoudre ! 
non des cultures, pour redonner à nos contemporains le goût de l'universelle valeur de leur culture et de ses interrogations ${ }^{22}$... Au risque (salvateur) de briser $c e$ consensus dont nous avons essayé de montrer les sous-entendus.

Pierre Monzani*.

* Ce texte est bien évidemment écrit à titre personnel et en aucun cas en tant que responsable du Centre international de synthèse.

22. Voir, sur ce plan, des ouvrages dont le succes et l'aspect combatif n'excluent nullement l'intérêt, comme ceux de George StEIner, La Culture contre l'homme, Paris, Le Seuil, 1973, d'Alain Finkielkraut, La Défaite de la pensée, Paris, Gallimard, 1987 et d'Allan Bloom, L'Ame désarmée, Paris, Julliard, 1987. 